\title{
About One of the Approaches for the Research of the Stress-Strain State of a Flange Connection with a Seal Made of an Alloy with Shape Memory
}

\author{
Andrey Boikov ${ }^{1, a}$, Lubov Mironova ${ }^{1, b^{*}}$ and Sergey Shishkin ${ }^{1, c}$ \\ ${ }^{1}$ Moscow Aviation Institute (State National Research University), 4, Volokolamskoe sh., \\ Moscow, 125993, Russia \\ aa.boickov@yandex.ru, bmironova_lub@mail.ru, cssshisha@yandex.ru
}

Keywords: Elastic - Plastic Problem, Materials with Shape Memory, Multilayer Ring, Clutch

\begin{abstract}
Design features of a flange connection with a seal made of an alloy with shape memory and the most commonly used methods of researching of the stress-strain state of such connections are estimated. Alternative approach for stress-strain state analysis is proposed, it is based on modeling of the contact zone of the sealing surfaces by means of an equivalent gap between the layers, the value of which changes during axial compression of the multilayer ring and goes into tension. Formulas for determining of contact stresses at the border of layers, which take into consideration the variable physical and mechanical properties of the materials of each layer are presented.
\end{abstract}

\section{Introduction}

Currently, a lot of practical experience has been accumulated in the sphere of design and exploitation of various constructions of flange connections.

Among many constructions, flange connections with a shape memory alloy coupling are promising, which allow to connect and produce pipelines with spatial branches or a large length. Construction of connection of two flanges by means of clutch, made of an alloy with shape memory, is presented in the figure 1 (a). Such connections are called thermomechanical connections.

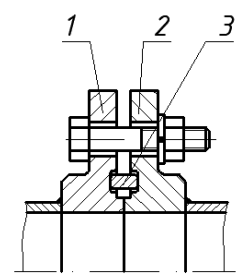

a)

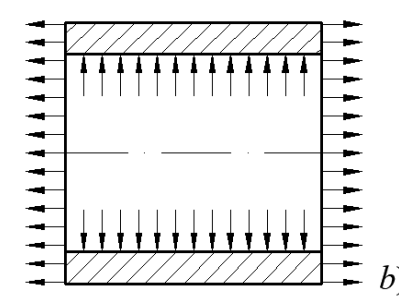

b)

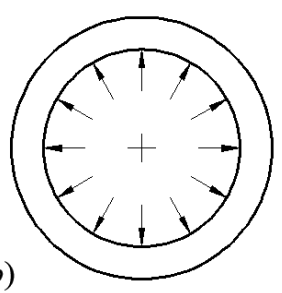

Fig. 1. Flange connection: $a$-construction; $b$-analysis model; 1, 2 -flanges, 3 - seal.

Thermomechanical connections of pipelines are a traditional and sufficiently proven field of application of shape memory alloys in terms of production. High operational characteristics and wide technological capabilities have led to the mass use of these connestions in shipbuilding, nuclear and aerospace engineering. The research of the strength of such connections is an urgent problem in ensuring operational reliability and issues of prolonging of the life of technical units. 


\section{Problem statement}

Flange connection construction with shape memory alloy coupling is estimated.

Flange connection consists of flanges 1 and 2, connected to the edge of pipes by means of welding. There are grooves, maiden on the end face of flanges. There is seal 3, mounted in it, made of alloy with shape memory. End faces of the grooves are bearing for the seal.

During the tightening of the bolts, the flanges converge in axial direction. Bearing surfaces of the grooves press on the seal, compressing it. Material of the seal deforms and expands radially under the action of compressive forces, filling the groove cavity. Contact stresses appear at the contact borders of the flange and seal surfaces in the radial and axial directions. The proposed model of the seal is a ring. Therefore, in many cases, well-known equations of the theory of axisymmetric deformation of rings are used for analyze it's stress-strain state, taking into account plastic deformation at small displacements, Fig 1 (b), [1-3].

The proposed formulas for determining the main stresses in the elastic and plastic zones of the analysis model are functions of the radius only [2], and do not depend on the physical and mechanical properties of materials. For our case, the elastic modulus of the flange and seal materials is different. In addition, the material of the seal has a shape memory effect. The phenomenon of pseudoelasticity is of the greatest interest here, in which plastic deformation, reaching tens of percent, is restored after the load is removed [4].

The engineering analysis of contact stresses in the structures of thermomechanical connections is based on modeling of the thermodeformation loading of a clutch, made of an alloy with shape memory by means of thermomechanical diagram [5]. It is a nonlinear dependence of the reactive return stresses on the magnitude of the under-recovery deformation.

However, the resistance of the pipe during forming is the cause of incomplete restoration of the original dimensions of the clutch during manufacturing, which leads to a contact load in the sealed joint.

Here, modeling of the stress-strain state based on the representation of the contact zone of the sealing surfaces by means of equivalent gap can be quite an acceptable way. We suppose, that the bolts are tightened and material of the seal has filled the groove cavities under the action of a compressive normal force. Then a multilayer ring can be used as analysis model, figure 2 .

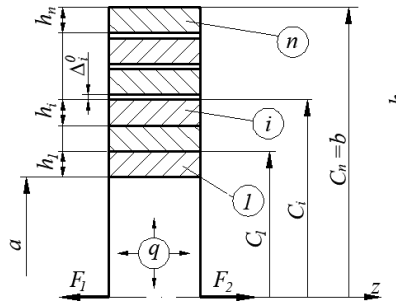

a)

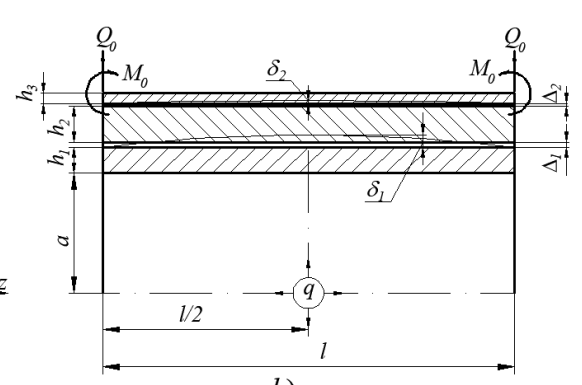

b)

Fig. 2. Multilayer ring: $a$-number of layers is $n$; $b$-number of layers is 3 .

The problem statement is formulated as follows. We suppose, that there is a complete multilayer ring in the disk with $n$ number of layers, and the number of layers is not limited. The ring is under the action of internal pressure $q$, axial force $P_{z}=q \pi a^{2}$ and under heating conditions $T=T(r)$ provided by the thermomechanical connection technology, Fig. 2 (a). There are gaps or tights between the layers before loading, which are small compared to the thickness of the minimum layer of the construction. 
Following the work [6], it is required to determine the stresses and contact pressures at the boundary of the ring layers, taking into consideration micro-gaps and micro-dimensions of surfaces under given initial conditions.

From the point of view of the engineering approach, all the considered layers in the form of mating surfaces refer to smooth cylindrical conjugations consisting of enclosing and encompassing smooth cylindrical surfaces. These interfaces are provided by the presence of gaps or tightnesses, which determines the freedom of their relative movement or the degree of resistance to mutual displacement during the thermomechanical connection.

\section{Basic approach and method description}

Let's denote the gaps or tightnesses which have place before loading in the multilayer structure $\Delta_{i}{ }^{0}$, and we assume that the gap corresponds to a positive value of $\Delta_{i}{ }^{0}$, the tightness - to negative one. The thickness of the layer is determined by the value $h_{i}$, and the layer itself is formed as a segment $c_{i}-c_{i-1}, i=(1,2,3)$.

Under the influence of internal pressure, the layers work in conditions of mutual contact, as a result of which, a contact pressure $p$ is formed between them. In this case, the micro-dimensions of the surface layer deform and move in the radial direction. The magnitude of such a displacement $\Delta_{p}$ is a function of the contact pressure and it is expressed by the dependence

$$
\Delta_{p}=\Delta_{p}(p)
$$

The surface layer has roughness, the magnitude of which depends on the manufacturing technology of the product. When rough surfaces come into contact, contact is formed between them at separate points. If there is a temperature effect during the manufacturing process, then a thermal contact resistance $R_{K}$ arises, the value of which is determined by the ratio

$$
R_{K}=R_{K}(p)
$$

The values (1) and (2) are determined experimentally, so we consider them known.

The stress-strain state of the ring is described by the equilibrium equation:

for the highlighted element of the ring

$$
r \frac{d \sigma_{r}}{d r}=\sigma_{t}-\sigma_{r}
$$

for the highlighted segment of the ring:

$$
\int_{a}^{b} \sigma_{z} r d r=\frac{q a^{2}}{2}
$$

where $\sigma_{t}, \sigma_{r}, \sigma_{z}$ are circular, radial and axial stresses, correspondingly.

In the case of thermal action, the equation of state of any point on the surface of the ring can be expressed by the following relation:

$$
\varepsilon_{r}=\frac{1}{E\left(c_{i}\right)}\left[\sigma_{r}-v\left(\sigma_{t}+\sigma_{z}\right)\right]+\alpha\left(c_{i}\right) T,
$$


where $E\left(c_{i}\right)$ is the modulus of elasticity; $v$ is the Poisson's ratio; $\alpha\left(c_{i}\right)$ is the coefficient of thermal expansion of each layer of the material. We consider the case of a plane deformation when $\varepsilon_{z}=$ const. Let 's write down the equation of thermal conductivity for a multilayer cylinder with stationary heat exchange in the following form

$$
\frac{d T}{d r}=-\frac{\bar{Q}}{2 \pi r}\left[\frac{1}{\lambda\left(c_{i}\right)}+\sum_{i} R_{K}\left(p_{i}\right) c_{i} \cdot \delta\left(1-\frac{c_{i}}{r}\right)\right],
$$

where $Q$ is the heat flux, acting in a unit of cylinder length; $\lambda\left(c_{i}\right)$ is the thermal conductivity coefficient of each layer of material; $\delta$ is the Dirac delta function.

If the deformations are compatible, radial displacement and relative radial deformation are expressed by the equalities [7]

$$
\begin{aligned}
& \frac{d u}{d r}=\varepsilon_{r}-\sum_{i}\left(\Delta_{i}^{0}+\Delta_{p}\left(p_{i}\right)\right) \cdot \delta\left(1-\frac{c_{i}}{r}\right) . \\
& \frac{d \varepsilon_{\theta}}{d r}=\frac{\varepsilon_{r}-\varepsilon_{\theta}}{r}-\frac{1}{r} \sum_{i}\left(\Delta_{i}^{0}+\Delta_{p}\left(p_{i}\right)\right) \cdot \delta\left(1-\frac{c_{i}}{r}\right) .
\end{aligned}
$$

Here $\varepsilon_{r}, \varepsilon_{\theta}$ are radial and circumferential deformations; $p_{i}=\left|\sigma_{r}\left(c_{i}\right)\right| ; \sigma_{r}\left(c_{i}\right) \leq 0$.

By integrating the system of equations $(3),(6)-(8)$ we obtain

$$
\begin{aligned}
& \sigma_{r}=-q \psi_{1}\left(\frac{a}{r}\right)+\sigma_{\theta}^{0} \psi_{2}\left(\frac{a}{r}\right)+\frac{\bar{Q} E\left(c_{i}\right) \alpha\left(c_{i}\right)}{(1-v) 4 \pi \lambda\left(c_{i}\right)} f_{r T}(r, \bar{p})-\frac{E\left(c_{i}\right)}{1-v^{2}} f_{r \Delta}\left(r, \bar{p}, \bar{\Delta}^{0}\right) ; \\
& \sigma_{t}=-q \psi_{2}\left(\frac{a}{r}\right)+\sigma_{t}^{0} \psi_{1}\left(\frac{a}{r}\right)+\frac{\bar{Q} E\left(c_{i}\right) \alpha\left(c_{i}\right)}{(1-v) 4 \pi \lambda\left(c_{i}\right)} f_{t T}(r, \bar{p})-\frac{E\left(c_{i}\right)}{1-v^{2}} f_{t \Delta}\left(r, \bar{p}, \bar{\Delta}^{0}\right) ; \\
& \sigma_{z}=\sigma_{z}^{0}+\frac{\bar{Q} E\left(c_{i}\right) \alpha\left(c_{i}\right)}{(1-v) 4 \pi \lambda\left(c_{i}\right)} f_{z T}(r, \bar{p})-\frac{v E\left(c_{i}\right)}{1-v^{2}} f_{z \Delta}\left(r, \bar{p}_{,}, \bar{\Delta}^{0}\right) ; \\
& T=T_{0}-\frac{\bar{Q}}{2 \pi \lambda\left(c_{i}\right)} f_{z T}(r, \bar{p}) .
\end{aligned}
$$

where $f_{r T}, f_{\theta}, f_{z T}$ are radial, circular and axial functions depending on the temperature distribution, the contact pressure between the layers and the thermal resistance, determine the name from article [7].

Expressions (9) - (12) use the following notation: vector of the contact pressure value is $\bar{p}=\left(p_{1}, p_{2}, \ldots, p_{n-1}\right)$; vector of initial gaps between cylinder layers is $\bar{\Delta}=\left(\Delta_{1}, \Delta_{2}, \ldots, \Delta_{n-1}\right) ; \sigma_{t}{ }^{0}$, $T_{0}$, are stress and temperature at points on the inner surface of the cylinder.

The boundary conditions on the inner and outer surfaces of the cylinder are formulated as follows, when $r=a ; \sigma_{r}=-q ; r=b ; \sigma_{r}=0$, then 


$$
\sigma_{t}^{0} \psi_{2}\left(\frac{a}{b}\right)-q \psi_{1}\left(\frac{a}{b}\right)+\frac{\bar{Q} E\left(c_{i}\right) \alpha\left(c_{i}\right)}{(1-v) 4 \pi \lambda\left(c_{i}\right)} f_{r T}(b, \bar{p})-\frac{E\left(c_{i}\right)}{1-v^{2}} f_{r \Delta}(b, \bar{p}, \bar{\Delta})=0 .
$$

Substituting the values of $r=c_{i},(i=1,2,3)$ into equation (9), we obtain a system of nonlinear equations regarding to the values of the contact pressure between the layers

$$
p_{i}=q \psi_{1}\left(\frac{a}{b}\right)-\sigma_{t}^{0} \psi_{2}\left(\frac{a}{b}\right)-\frac{\bar{Q} E\left(c_{i}\right) \alpha\left(c_{i}\right)}{(1-v) 4 \pi \lambda\left(c_{i}\right)} f_{r T}(b, \bar{p})+\frac{E\left(c_{i}\right)}{1-v^{2}} f_{r \Delta}(b, \bar{p}, \bar{\Delta})=0 .
$$

The solution of the system of equations (13) and (14) has mathematical difficulties. Therefore, to determine the contact pressure between the layers, we use the well-known formula for elongating of the radius of the cylinder $\delta$ under the action of internal pressure from the work [8], which forms the contact pressure between layers 1, 2, 3 at the border $c_{i}$, Fig 2 (b) Taking into consideration the thermal expansion of the material under thermal action, we believe that [6]

$$
\delta_{i}=\frac{q a^{2}}{E_{i} \sum_{i=1}^{n} h_{i}}\left(1-\frac{v}{2}\right)+\alpha_{i(T)} T h_{i} ; p_{i}=\frac{\left(\delta_{i}-\Delta_{i}\right) E_{i} h_{i}}{a^{2}\left(1-\frac{v}{2}\right)} \text {. }
$$

\section{Conclusion}

The proposed approach makes it possible to evaluate the stress-strain state of a flange connection with a seal made of shape memory alloy and determine the maximum contact pressure between layers with acceptable accuracy in engineering practice. According to the strength criterion, the calculated values of the contact pressure should not exceed the permissible values of the contact stress.

\section{References}

[1] N.I. Bezuhov, Bases of the theory of elasticity, plasticity and creep, Vysshaya shkola, Moscow, 1968.

[2] N.N. Malinin, Applied theory of plasticity and creep, Mashinostroenie, Moscow, 1975.

[3] R. Hill, The Mathematical theory of plasticity, at the Clarendon press, Oxford, 1950.

[4] V.A. Lihachev, S.L. Kuz'min, Z.P. Kamenceva, Shape memory effect, Leningrad, LGU, 1987.

[5] S.V. Shishkin, Experimental determination of the generalized thermomechanical diagram of shape memory alloys under axisymmetric bending, J. Industrial Laboratory 60 (2) (1994) 124125.

[6] L.I. Mironova, A.S. Ivanov, Effect of defects on technological thermal stress state of multilayer cylindrical structure, J. Engineering \& Automation Problems, 2 (2013), 121-125.

[7] P.G. Pimshtejn, V.N. Zhukova, Calculation of stresses in a multilayer cylinder taking into account the peculiarities of the contact of the layers, J. Strength problems 5 (1977) 71-75.

[8] Timoshenko S, Vojnovskij - Kriger S. Theory of plates and shells.MeGraw-Hill Boor Company, INC, New-York, Toronto, London, 1959. 